\title{
The Design of Headword in the Online Shopping's Advertisement
}

\author{
Cuiyan Niu \\ Hebei Academy of Fine Arts, Shijiazhuang Hebei, 050700, China
}

Keywords: Online shopping, Headword, Design.

\begin{abstract}
In the rapid developing of the network technology in China presently, online shopping has become a major favored shopping approach by most people, even has evolved into a new way of life. The headword in online advertising is not only to tell shopping information to the consumer, but also to let the consumer have mentality resonance and spiritual enjoyment. Let some of the flat's visual elements into the design of headword in the online shopping's advertisement, which can achieve some unexpected effectiveness. It is worth us to struggling and exploring.
\end{abstract}

\section{Introduction}

The headword design of the online advertisement is generally based on the basic print by arrangement and combination, decoration, change, and processing. This can get rid of the basic font's serious feeling. According to the topic, we can combine the thoughts and the rich imagination to organize font's appearance by more vivid, flexible method, which would form unexpected and creative design. It also can enhance the spirit meaning and infectivity of words, and the effect of visual communicating effect.

\section{The title in the online advertising, the common choice of font, size, spacing and row spacing}

The title in the online advertising needs a design which is not only reflecting the theme's image, but also have a creative modeling design. In the first place, we should choose the appropriate print as the basis of the design. Then put the font into properly spacing of word gap and row. At last, do the change of the design. This is the precondition of headword design. Each type has its own characteristics and cultural temperament. The different products of online shopping have its own connotation and brand image, which requires the designer to accurately transmit font's selection, design and contents to the consumer.

\section{The font's selection}

The font's selection depends on practicability and creativeness. Creativeness is to break the routine, cooperate with their title and choose the corresponding font. For example, the female cosmetics use feminine and comely words. Practicability is the core of the font selection. We do not need gorgeous and flamboyant font. And we just want consumers to feel comfortable, and reflect the theme perfectly with its own design features. This needs us to match the type of online advertising title and font. And the design style can be divided into the following kinds:

Gentle and graceful style

This kind of font is dignified, graceful and elegant. The font's line is fluent, and structure is rigorous and upright, such as song typeface, coarse qian typeface, founder typeface of black simplified, etc. This type is applicable to female cosmetics, bags, shoes, clothes, daily provisions, service advertisement title in online shopping.

Fun and lively style 
This kind of font is vivid, lovely, lively, leap feeling. The color is bright and colorful. This type has the distinct rhythm feeling and metrical sense, which gives vibrant visual perception, such as founder typeface of simplified children, playful typeface, mangle and so on. This type is applicable to the maternal and infant supplies, leisure food, sports and other advertisement title in online shopping. Concise and straight style

This kind of font is suitable for being the headline. This style is without redundant adornment, is strong, tall, straight, founder, simple concise and bright, eye-catching and prominent, and has strong contemporary feeling. Therefore, this style is suitable for most kinds of online advertising text design, such as the goods total title, machinery, electronic products. The font usually uses wide variety of typeface, han's standard typeface, lingxin typeface, coarse qian typeface, founder simplified typeface, black gauge body typeface, etc.

Primitive and refined style

This font is Chinese calligraphy font. It is vigorous, has deep cultural atmosphere. It is full of classical, elegant, magnificent, charming, elegant atmosphere. That brings a kind of psychology sense which is nostalgic, cultural, tasteful, and trust. If combine the calligraphy font and print font together perfectly, it will be another kind of fashion style which is opponent to the primitive and refined style.

\section{The size of font}

The size of font depends on two aspects. One aspect is the comparison relationship among the different level of headword's combination. The size of headword should be most striking in the combination. Another is the subtitle which is the explanation of the heading. When people are interested in the headline, they will pay attention to the subtitle. So the size of subtitle should be smaller than the headline. The other aspect is that the font of the headline should distinguish obviously by using different style of typeface and size, such as style, type, thickness and so on. These factors are also design in different marked degree of the size according to the primary and secondary relationship.

\section{The font spacing and row spacing}

In common, it refers to the numerous words in the paragraphs. In the headline of advertisement is different from the paragraph.

In order to making the headline outstanding, the combination is rigorous, integrity. Each word should be very compact to achieve the overall unity of the text structure.

Different type of font's characters occupies different proportion of space. Such as the regular script character occupy small rate of space. If the font spacing is too wide, it will be scattered on the vision. Black typeface and Song typeface occupy bigger rate of the space, so the bigger font spacing will be more comfortable. The rows in the headline should follow the principle of the row spacing greater than the font spacing when you want to get the different level, clear proportion, easy to read and recognize. If you have special topic design, you also could tighten the row spacing.

\section{Structural change in the stroke}

The basic Chinese characters is stroke, which can do the facile and diversity design by using the abstract horizontal stroke, vertical stroke, point stroke, left-falling stroke, right-falling, hook stroke. So the font design is according to the different strokes, meaning, and shape. Usually this can be divided into the following kinds:

\section{Using the unified form elements}

Generally the horizontal stroke and vertical stroke are the main stroke. Their form only changes in the length and the thickness. The design is always changing in the point stroke, left-falling stroke, right-falling, hook stroke. When design the stroke shape, we find the regular of its change. Then other words in the headline will change according to this regular, so that the whole headline demonstrates a consistency, integrity and unity. It should avoid excessive complex and too many forms of the words in the headline. Otherwise it will cause a dazzling vision, even losing the basic recognition. 


\section{Joining the individual different elements in the unified form}

This method is based on the unified font style. In the first place, it should ensure the overall unity of the headline. Secondly, it makes the changing design of the individual characters in accordance with the theme. Compare with the first method, this is more outstanding which can achieve the effect by stressing the meaning of the subject among some individual elements with pictures and words.

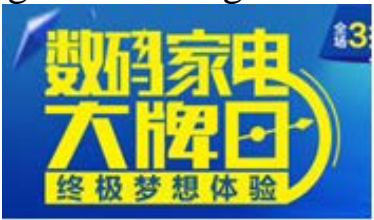

Picture 1-1

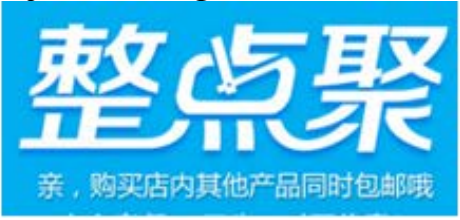

Picture 1-2

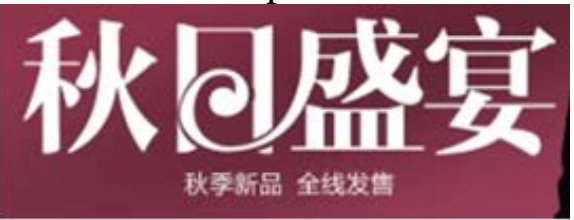

Picture 1-3

In the headline of "the brand of digital appliances' day" in Picture 1-1, the code, electricity, and day respectively use lightning and the clock dial modeling replace some strokes. It is not only enriching the image, but also reflecting the theme of the connotation. Picture 1-2 uses the same way to demonstrate the "point" by the form. The theme is very obvious. Picture 1-3 uses Arial as the printing matrix. It designs the strokes of "sheng" skillfully by flower clip to replace the strokes of the "ri". That makes the whole headline being elegant, and without losing lively and playful.

\section{Elongating or shortening the font strokes}

A. Elongating or shortening the whole combination headline

This method makes the overall title elongating directly which is easy to design and read, and keeps the recognition. But it needs to pay attention to the moderation of elongating about the aesthetic. If the combination is too long that would lose the beauty. Elongating too long will change the structure of the headline's balance, so some fonts are suitable, and some are not suitable.

B. Stretching or shortening (missing) individual stroke

Under the whole situation, we can stretch the individual strokes naturally and shorten strokes with matching the theme. We need to pay attention to the show the combination relationship which is overall harmony and comity. When use this method in design, we should select the suitable words to elongate or shorten. Otherwise it will be ugly, and its recognition is also weakened.

\section{Strokes sharing}

We can regard the stroke as the line, then ingeniously combine the stroke's commonness to be its own word and mutually connected to form a new shape. Borrowing the stroke should be natural and reasonable, do not be farfetched and stiff. It is better to keep the recognition, and be small connection, breaking and changing to get the big effect. This requires us to find out the useful stroke to slightly change the length to create opportunities.

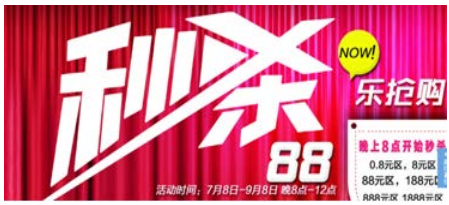

As shown in the picture: in the two words of "spike", it extended the stroke of "kill" and shared with the stroke of "seconds" .this stroke instantly demonstrate the vigor in a perfect way in front of us.

\section{Representational change}

The original of Chinese characters is pictograph which uses the shapes of physical objects to represent its name, or uses the figure instead of the whole individual word, or use the specific image instead of Chinese characters or strokes and components. This design will use the figure to demonstrate the meaning, and achieve the visual effect which combines the picture and word equitably. No matter which kind of expression requires complying with the basic principles of Chinese characters that is the recognition after designing. 


\section{Expressing directly - use the specific image to statement the word's meaning}

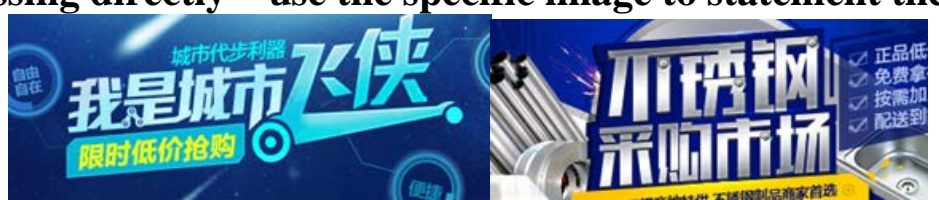

Picture 1-4

Picture 1-5

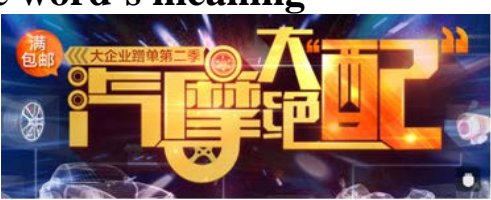

Picture 1-6

Picture 1-4, Picture 1-5 and Picture 1-6 combine the specific image of good's selling point and headline words ingeniously. It is not only highlighting the theme, but also conveys the nature of commodity. This achieves the effect of using the image to demonstrate meaning authentically.

\section{Expressing indirectly}

We can use the relative related symbols or images instead of strokes or radicals with the aid of the artistic conception by word or phrase, thus indirectly show the intension of the word.

\section{The decorative font}

When design the headword, we can add graphics in the internal and external of the stroke, or extend the stroke to connect the figure. Using this art designing skill for decorating can render the headline's form and rich the connotation. On the basis of maintaining the shape and the readability of the text, it is easier for people to understand the content directly or indirectly. These decorative skills should combine with decorative fonts, and become a unified and harmonious entirety. Thereby, to enrich the artistic image, expand its artistic expressing, and ultimately reflect consumer's purchasing psychology.

Decorating skill is various, and has its own characteristics. Taking the headword in the online shopping advertisement as example, we can divide decorative skill into three factors: the demonstrating of the headword's space, the inside and outside decorating of the word and the charming of handwriting font.

\section{Space performance}

Using the shadow, embossment, perspective, overlap and other forms of expression way to show the three-dimensional effect of the words, and combine with the different materials of font to achieve eye-catching effect.

\section{The inside and outside decoration of the character}

The full space decoration in the text strokes

Picture 1-7 - 9 shows that the strokes are filled with texture, graphics, images and other decorative patterns which signify the theme, and transmit the theme more precise.

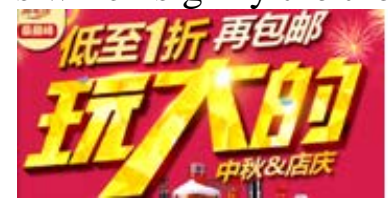

Picture 1-7

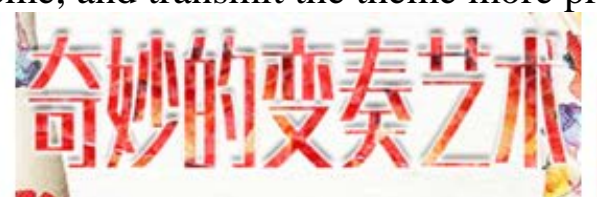

Picture 1-8

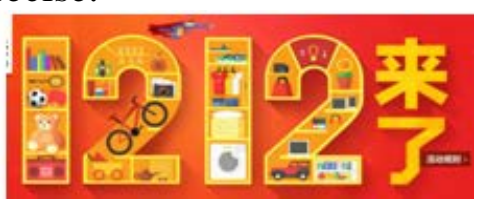

Picture 1-9

The surrounding decoration of the font

As shown in the Picture 1-10 - 13, it tells us more direct and clear connotation of the theme in vision according to the meaning of the headwords information with exaggerated graphics. Picture 4-62 make the background to white. It is not only highlighting the font, but also list the major product among the surrounding environment (all kinds of toys). 


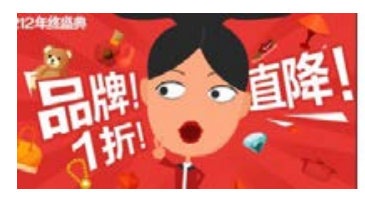

Picture 1-10

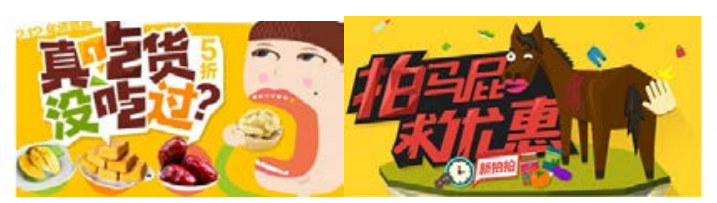

Picture 1-11

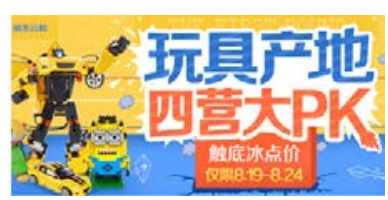

Picture 1-13

\section{Handwriting}

Handwriting has no rules, and has its special artistic and vivid. Compared with printed, handwriting is more friendly, free, rich and varied. As young children's handwriting, it gives a person naive sense, and can be easy recognized. The handwriting contains the calligraphy font which is full of traditional history and cultural atmosphere. Its national characteristic is prominent. In common, the local traditional characteristic product and various kinds of packing design use the young children's handwriting in the online shopping advertisement.

\section{Calligraphy}

As shown in Picture 1-14 - 16, the calligraphy is free, powerful, generous and fluent. The calligraphy contains full of rich history, culture and national characteristic.

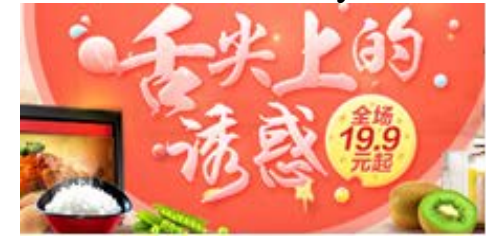

Picture 1-14

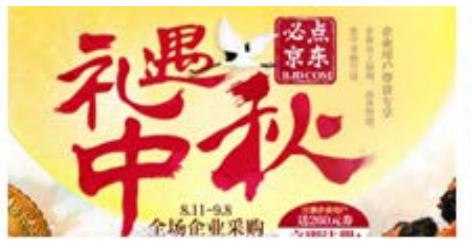

Picture 1-15

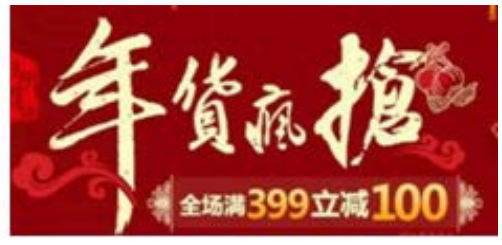

Picture 1-16

\section{Handwriting}

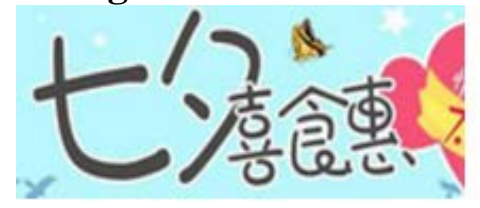

Picture 1-17

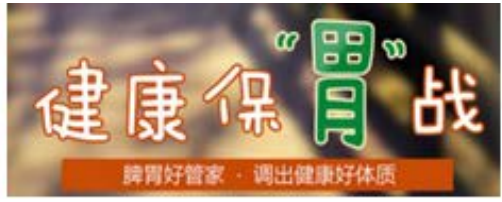

Picture 1-18

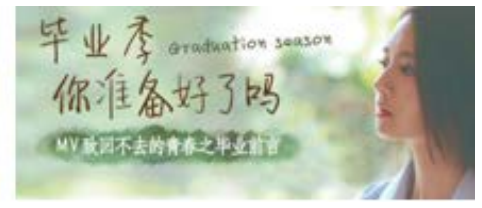

Picture 1-19

As shown in the Picture 1-17 - 19, different types of handwriting have individual styles. It is kind and free, such as "graduation season, are you ready?" .The font is comely. Even we can feel wistful by the handwriting font that is expressed very clear.

The most important point is the character design should be novel, unique and beautiful to make the headline of the advertisement to be mindful. Then, the character of the headline is able to concisely and directly reflect the needs of consumers. So the advertisement can attract different types of consumers who have different needs. Thirdly, the headline of the advertisement should emphasize the theme. It needs to use more vivid modeling to modify the word, to use prominent expression of verbal semantics, so that makes the headline more vivid and to use the shape to express the meaning. The last is to simplify the word. Utilizing the limited words to fully express the semantics and highlight the selling point to make the headline more concise, penetrating, and accurate. This way can stimulate the desire of purchasing.

\section{References}

[1] Addison’s Quotes. Shanghai Social Science Press, 1980:1.

[2] Hopkins’ Quotes. Humor Wins the Favor of Others in Arbitrary Moment. China Press, 2001:13.

[3] The Font Design, Shen Zhuoya. editor.Higher Education Press, 2005:7.8.9. 
[4] The Font Design, edited by Zhang Aimin, Zhou Zan. China Youth Press, 2009:8.9.

[5] Advertising Creativity and Performance, Rao Dejiang, editor, The Radio and Television University of China press, 2001:11.13.

[6] Modern Advertisement Design, Dong Xiaolong,etc. Hebei Arts Publishing Company, 2007:13.

[7] World classic Advertising Case analysis, Zhang Jinhai, editor, Wuhan University Press, 2000

[8] William Ahrens, Contemporary Advertising, Translated by Ding Junjie and Zhang Xi. Huaxia Publishing Company, 2001 